\title{
Tuberculosis Treatment Outcomes and Associated Factors Among Tuberculosis Patients in Bule Hora General Hospital, Southern Ethiopia, 2021: Retrospective Cross-sectional Study
}

Dawit Galgalo ( $\square$ dawitgalgalo04@gmail.com )

Bule Hora University

\section{Research Article}

Keywords: Bule Hora Hospital, Tuberculosis, TB Treatment Outcomes, Ethiopia

Posted Date: January 19th, 2022

DOI: https://doi.org/10.21203/rs.3.rs-1273888/v1

License: @ (i) This work is licensed under a Creative Commons Attribution 4.0 International License. Read Full License 


\section{Abstract \\ Background}

In Ethiopia tuberculosis is the leading causes of morbidity, the incidence of all forms of TB in the Ethiopia was $379 / 100000$ population which is the second on the Africa continent. There are high discrepancies in the result of treatment outcome being reported from the different region of the country with frequent poor treatment outcomes particularly from the southern Ethiopia. Thus, this study aims to assess tuberculosis treatment outcomes and associated factors among tuberculosis patients in Bule Hora general hospital, southern Ethiopia

\section{Methods}

Facility based retrospective cross-sectional document review of 349 patients was conducted to assess the TB treatment outcome and associated factors for TB patients' document registration. Data was entered into the computer using Epi-data software version 3.2 and exported to SPSS software version 22 for analysis. Bivariate and multivariate analysis with $95 \%$ confidence intervals were employed to infer associations between the independent and dependent variables.

\section{Result}

The documents of 349 tuberculosis patients were reviewed. Among this patients, $87.9 \%$ were successfully treated whereas $12.1 \%$ of the patients were unsuccessfully treated. From this, $53.4 \%$ and $34.5 \%$ patients were cured and completed the treatment, respectively, whereas $3.7 \%, 5.8 \%$, and $2.6 \%$ patients were died, treatment failure, and defaulted, respectively during the follow-up. The tuberculosis patients who were screed positive for HIV/AIDS were $86 \%$ less likely to had a successful TB treatment outcome than HIV/AID negative patients.

\section{Conclusion}

The TB treatment outcome for the present study was $87.9 \%$, which was lower than national achievement in $2017(96 \%)$ and global target $(>=90 \%)$ which required improvement. The higher unsuccessful TB treatment outcomes among HIV positive patients suggests the need to strengthen adherence education and supervision.

\section{Highlights}

\section{What is already known on this topic?}

- Ethiopia is one of the 30 highest TB burden countries in the world 
- The Ethiopian national case notification rate for all forms of TB was $67.3 \%$, with a cure rate of $77.9 \%$.

- The WHO targets for TB control with a $70 \%$ case notification rate and $85 \%$ cure rate

\section{What this research adds?}

- The TB treatment outcome for the present study was $87.9 \%$, which was lower than national achievement in 2017 (96\%) and global target (>=90\%) which required improvement.

- Among $12 \% .1$ of unsuccessful TB treatment outcomes, $6.8 \%$ of them were HIV positive

\section{Introduction}

Globally one-third of the world population is infected with TB. Every year TB affects 10 million people. Every 15 seconds, one person dies from TB comprising more than 2 million death annually. The Africa continent consists of $28 \%$ of world cases. In Ethiopia TB is the leading causes of morbidity, the incidence of all forms of TB in the Ethiopia was 379/100000 population which is the second on the Africa continent. Tuberculosis was also the leading causes of hospital admission and the second causes of death, affecting more than 76000 people annually [1].

In Ethiopia, the prevalence, incidence, and morbidity from TB are currently estimated at $200 / 100,000$ people, $207 / 100,000$ people, and $33 / 100,000$ people, respectively. This indicators show that the TB burdens in Ethiopia is still enormous and suggest that the national TB control program should be functioning very well to achieve a significant reduction in morbidity and mortality resulting from TB [2].

Ethiopia is one of the 30 highest TB burden countries (HBC) in the world. In 2015, the national case notification rate for all forms of TB was $67.3 \%$ with cure rate of $77.9 \%$. One of the studies conducted in Oromia region, Ethiopia, revealed that the TB case notification rate was $65.4 \%$. unfortunately, this achievement is lower than the WHO targets for TB control with a $70 \%$ case notification rate and $85 \%$ cure rate $[1,3]$.

Even though there are some improvements in terms of TB treatment success and case detection rate, the patient outcomes of TB treatment have been different in the different parts of Ethiopia. The study conducted on the treatment outcomes of tuberculosis patient in Metema hospital indicated that among 2970 patients who initiated ant-TB treatment indicated that only $50.3 \%$ of patients completed treatment. The overall death, defaulter, and failure rate among the study participants were $3 \%, 3.3 \%$ and $0.7 \%$ respectively [3-4].

Despite a number of the study conducted regarding tuberculosis treatment outcomes among hospital in the countries, there is still no data that indicated the characteristics of tuberculosis treatment outcomes particularly in the Bule Hora General Hospital. Thus, this study aims to assess tuberculosis treatment 
outcomes and associated factors among tuberculosis patients in Bule Hora general hospital, southern Ethiopia. The results of this provide appropriate information for the different health institution as well as for concerned stake holders in the area and elsewhere. Additionally, the result of this study provide important input for policy makers, health planners, and decision makers and used as baseline data for researchers who want to conduct the study this area.

\section{Methods And Materials}

\section{Study Area and Period}

Bule Hora general hospital, which is found at $470 \mathrm{~km}$ from the capital Addis Ababa, is located in the West Guji Zone, Oromia region, South Ethiopia. The hospital has a capacity of 44 beds, providing services for over 1500 patients per year. The hospital has a direct observetion theraphy(DOT) clinic where TB patients are treated and monitored according to the National Tuberculosis and Leprosy Control program guidelines. The study was conducted on the TB treatment outcome and associated factors among TB patients at Bule Hora General Hospital from Janury1-30, 2020.

\section{Study Design}

Institution based retrospective cross-sectional document review was been conducted to assess the treatment outcome of TB patients and the associated factors of TB patients' documents registered from January 1, 2015, to July 2019 at Bule Hora General Hospital.

\section{Population}

Source population: The documents of all TB patients who were registered in the hospital from the 1st of January, 2015, to July 2019.

Study population: The documents of the randomly selected TB patients who were registered in the Blue Hora General hospital from the 1st of January, 2015, to July 2019.

\section{Inclusion and Exclusion Criteria}

\section{Inclusion criteria}

The documents of the registered TB patients at Bule Hora General Hospital were included in this study.

\section{Exclusion Criteria}

However, registries in which treatment outcomes were missing and patients were transferred to other districts were excluded from the study. 


\section{Variables}

\section{Dependent variables}

Treatment outcomes are categorized into successful and unsuccessful treatment outcomes. Successful treatment outcomes included "cured" and "treatment completed" cases. Unsuccessful treatment outcomes included "treatment failure" cases, "defaulters," and patients who "died.

\section{Independent variables}

Socio-demographic factors: Sex, age, place of residence, place of residence, and year of treatment.

Clinical factors: Category of patient types of TB, Smear result, HIV status, ART initiated.

\section{Sample Size Determination}

For the first objective, the sample size was calculated by using a single population proportion formula based on the assumption of TB Treatment Outcomes in Metema Hospital of $65.3 \%$ (P) was used in this case [2].

Where;

- $\mathrm{P}=$ TB Treatment Outcomes (65.3\%).

- $Z$ = standard score corresponds to1.96.

- $d=$ margin of error 0.05 with a $95 \%$ confidence interval was used.

$$
\frac{\mathrm{z}^{2}(1-\alpha / 2) \mathrm{p}(1-\mathrm{P})}{\mathrm{d}^{2}}=\frac{(1.96)^{2} * 0.653 * 0.347}{(0.05)^{2}}=349
$$

Finally, the sample size of 349 obtained from the first specific objective was used, because it is the largest sample size estimated and would be sufficient for study.

\section{Sampling Technique}


Totally 349 TB patients documents reviewed from January 1st, 2015, to July 2019 , in the Blue Hora general hospital. The registries which met the inclusion criteria were selected through a systematic sampling technique. This sampling interval $(K)$ will be obtained by dividing the total TB patients by its sample size $(k=1869 / 940=1.99 \sim 2$. The 1 st sample was taken by lottery method from 1 ; then the data will be collected from the registration log book.

\section{Operational Definition}

Successful outcome: if the TB patient will be cured (negative smear microscopy at the end of the treatment and on at least one previous follow-up test) or completed treatment with resolution of symptoms.

Unsuccessful outcome: if the treatment resulted in treatment failure (remaining smear positive after 5 months of treatment), patients are defaulted (patients who interrupted their treatment for two consecutive months or more after registration), or patients died. According to the standard definitions of the National Tuberculosis and Leprosy Control Program guidelines of Ethiopia [5], the following definitions were used for treatment outcomes.

Cured: if the patient had finished treatment with negative bacteriological results at the end of the treatment.

Treatment completed: if the patient had finished treatment, but without bacteriological results at the end of the treatment.

Treatment failure: a patient who, while on treatment, remained smeary positive or became again smear positive at the end of five months or later after commencing treatment or a patient who was PTB negative at the beginning and turned out smear positive at the end of the intensive phase.

Defaulter: a patient who had been on treatment for at least 4 weeks and whose treatment was interrupted for 8 or more consecutive weeks.

Died: if the patient died from any cause during the treatment.

New case: a patient who had never had treatment for TB or had been on anti-TB treatment for less than four weeks.

Three types of TB will be considered in this study. These are smear positive pulmonary TB (PTB+), smear negative pulmonary TB (PTB-), and extrapulmonary TB (EPTB).

Smear positive pulmonary TB (PTB+): a patient who had at least 2 initial smear examinations positive for AFB by direct microscopy or one initial smear examination positive and culture positive or one initial smear positive and radiographic abnormalities consistent with TB. 
Smear negative pulmonary TB (PTB-): a patient who had three initial smear examinations negative for AFB and no response to the course of broad-spectrum chemotherapy and again 3 smear examinations negative by direct microscopy and radiographic abnormalities consistent with PTB and three initial smear examinations negative by direct microscopy but positive by culture and decision by a clinician to treat with anti-TB

extra pulmonary TB (EPTB): a patient who had TB in organs other than the lungs proven by one culturepositive specimen from extra pulmonary sites or histopathological evidence from a biopsy or TB based on strong clinical evidence for active EPTB and the decision by a physician to treat with anti-TB.

\section{Data collection tools and technique}

The data was collected by using a pretested structured data extraction format. The standard TB registry, laboratory findings, and monthly cohort follow-up form were reviewed to generate the required data. The standardized checklist was used including all important socio-demographic data, clinical characteristics (sputum smear, type of TB, patient type, HIV status, drug regimen, and treatment outcomes), laboratory findings, and follow-up data. Data were collected by four BSc. Nurses who have had training on comprehensive TB care and experience in data collection.

\section{Data quality assurance}

To improve the quality of the data, the questionnaire was pretested a week before the actual data collection time on a 5\% sampled unit in a Qarca hospital and modification was done accordingly. The supervisor and data collectors were trained for two days before data collection. The proper supervision of data consistency and completeness was undertaken throughout the data collection and analysis. The data collectors discussed the methods of data collection and the questionnaire will be checked daily for completeness during data collection.

\section{Data processing and analysis}

Data was entered into the computer using Epi-data software version 3.2 and exported to SPSS software version 22 for analysis. Appropriate descriptive statistics such as mean (with standard deviation), median (with interquartile range [IQR]), and frequency (with percentages) were used to describe the study population in relation to relevant variables. Bivariate and multivariate analyses with $95 \%$ confidence intervals were employed to infer associations between the independent and dependent variables. Binary logistic regression was used to calculate the crude odds ratio (COR) with a 95\% confidence interval. Each 
variable was entered into a logistic regression model to determine the presence of a statistically significant association with the outcome variable. Multicollinearity among selected independent variables was checked through variance inflation factor (VIF). The explanatory variables with a P-value $\leq$ of 0.2 in the bivariate analyses were included in the final multivariable logistic model to identify the independent predictors of TB treatment outcome. AP value $<0.05$ was considered statistically significant. The assumption on the fitness of goodness of the final model was checked by Hosmer and Lemeshow test.

\section{Ethical consideration}

This study was conducted in accordance with the research ethics of the University of Bule Hora. Ethical clearance was obtained from the institutional ethical review board of the University of Bule Hora. As this is a retrospective study, the consent of patients was not obtained. However, patient information has been handled anonymously with assuring confidentiality.

\section{Results}

\section{SOCIO-DEMOGRAPHIC STATUS OF THE STUDY}

In this study, the total 349 TB patient registration logbook of five years was reviewed. The reviewed document indicated that from the total 349 TB patients received anti-TB drugs, $52.90 \%$ were male whereas $47.10 \%$ of the patient were female. The mean ages of patients was 34 years with a standard deviation of 17.78 . from the total 380 patients, the majority (91.30\%) were pulmonary TB whereas $8.70 \%$ were extra-pulmonary TB. Among the total patients receive ant-TB drugs,93.20\% were HIV negative and $6.8 \%$ were HIV positive. (See table 1 below)

Table 1: Socio demographic status of TB treatment outcome and associated factors in Bule Hora General Hospital, Ethiopia 2021 


\begin{tabular}{llll}
\hline Variables & Categories & Frequency & Percent\% \\
\hline Sex & Male & 201 & 52.90 \\
& Female & 179 & 47.10 \\
\hline Residence & Urban & 243 & 63.90 \\
\hline Age Of Patient & Rural & 136 & 35.80 \\
\hline & $<20$ & 150 & 39.50 \\
\hline Year Of Treatment & $20-40$ & 177 & 46.60 \\
\hline & 2016 & 53 & 13.90 \\
\hline HIV Status & 2017 & 1 & 0.30 \\
\hline Patient Category & 2018 & 122 & 32.10 \\
\hline \multirow{2}{*}{ Types Of TB } & 2019 & 138 & 36.30 \\
\hline & Positive & 119 & 31.30 \\
\hline Smear Result & Negative & 354 & 6.80 \\
\hline & Pulmonary TB & 347 & 93.20 \\
\hline & Extra PTB & 33 & 93.70 \\
\hline
\end{tabular}

\subsection{Tuberculosis Treatment Outcome}

In the review of the document of 380 TB patients, $87.9 \%$ were successfully treated whereas $12.1 \%$ of the patients were unsuccessfully treated. From this, $53.4 \%$ and $34.5 \%$ of patients were cured and completed the treatment, respectively, whereas $3.7 \%, 5.8 \%$, and $2.6 \%$ of patients died, treatment failure, and defaulted respectively during the follow-up.

The binary logistic regression was carried out to determine the factors associated with TB treatment outcome. The variables which had significance at the level of $p<0.25$ in the simple binary logistic regression were entered into the multiple binary logistic regression model. Finally, the variables which were still significant at the level of $p<0.05$ in the final model were taken as factors that were significantly associated with the outcome variables.

The four variables which were significant in the first model were still significant in the finalmodel. Thus, the age of the patient, year of treatment, the status of HIV, and patient categories were the factors that were significantly associated with TB treatment outcome in the final model.

The patients between the age group of $20-40$ were $83 \%$ less likely to have a successful TB treatment outcome than the patients with an age group of fewer than 20 years $[A O R ; 0.17,95 \% \mathrm{Cl}(0.04,0.76)]$. The tuberculosis patients who were screed positive for HIV/AIDS were $86 \%$ less likely to had a successful 
TB treatment outcome than HIV/AID negative patients [AOR; $0.24,95 \% \mathrm{Cl}(0.08,0.70)$ ]

The patients who were registered for tuberculosis treatment in 2017 had four times successful TB treatment outcomes than the patients who were registered in 2019 [AOR; 4.19, 95\% Cl $(1.30,9.57)$ (see table 2 below)

Table 2: the factors that are significantly associated with TB treatment outcome using bivariate and multivariate logistic regression

\begin{tabular}{|c|c|c|c|c|c|}
\hline & & TB treatmer & t outcome & COR(95\% CI) & AOR(95\% \\
\hline variables & & Successful & Unsuccessful & & \\
\hline & & $\mathrm{N}(\%)$ & $\mathrm{N}(\%)$ & & \\
\hline Age of & $<20$ & $147(43.60)$ & $3(7.00)$ & $\mathrm{I}$ & $\mathrm{I}$ \\
\hline patient & $20-40$ & $156(46.30)$ & $21(48.80$ & $0.76(0.38,1.54)$ & $0.17(0.04,0.76)$ \\
\hline & $>40$ & $34(10.10)$ & $19(44.20)$ & $0.03(0.01,0.13)$ & $0.02(0.05,0.11)$ \\
\hline HIV status & negative & $321(95.30)$ & $33(76.70)$ & $\mathrm{I}$ & I \\
\hline & positive & $16(4.70)$ & $10(23.30)$ & $0.16(0.06,0.39)$ & $0.24(0.08,0.70)$ \\
\hline patient & new case & $323(95.80)$ & $33(76.70)$ & $0.14(0.05,0.34)$ & $0.31(0.10,0.92)$ \\
\hline category & $\begin{array}{l}\text { re } \\
\text { treatment }\end{array}$ & $14(4.20)$ & $10(23.30)$ & $\mathrm{I}$ & I \\
\hline year of & 2010 & $117(34.70)$ & $5(11.60)$ & $3.37(1.19,9.60)$ & $4.19(1.30,9.57)$ \\
\hline treatment & 2011 & $116(34.40)$ & $22(51.20)$ & $0.76(0.38,1.54)$ & $0.59(0.26,1.35)$ \\
\hline & 2012 & $104(30.90)$ & $15(34.90)$ & I & $\mathrm{I}$ \\
\hline
\end{tabular}

Abbreviations: AOR, adjusted odd ratio; CI, confidence interval; COR, crude odd ratio; HIV, human immunodeficiency virus

\section{Discussion}

This was a preliminary study conducted on the TB treatment outcome and associated factors in the Bule Hora General hospital on tuberculosis patients who were enrolled and had taken Ant- TB drugs from 2015 to 2019. In the study, the prevalence of successful TB treatment outcomes was $87 \%$. This finding was controversial than the national estimates of the Ethiopian Ministry of Health which indicates a $96 \%$ of TB treatment success rate [3]. This difference might be due to the extent of the difference in the study area and population, but the result of the current study was based on a single institution.

The result of this study is lower than the study conducted in Harar Hospital which showed that The overall rate of treatment success among the patients was 92.5\%[5] and higher than the study conducted at Woldia General Hospital [ 80.7\%], Metema Hospital[65.3\% ], Gimbi Town health facility [74\%], Afar Region [81.8\%][6 ] and Pakistan [66\%][4]. The difference may be due to the period and place the studies were conducted as well as the setting in which the studies had been performed. However, the result of current studies was similar to the study conducted in the Tigray region [87\%] [7] bale zone [87.8\%] had a successful treatment outcome. [8] 
The results of this study found that $3.7 \%, 5.8 \%$, and $2.6 \%$ of patients died, treatment failure, and defaulted respectively during the follow-up. This finding was nearly the same as the study conducted in Metema Hospital that indicated the overall death, defaulter, and failure rates among the study participants were (3.0\%), (3.3\%) and (0.7\%) respectively[9]. However, this finding is different from the study conducted in Pakistan that showed that $51.2 \%$ were defaulters, $22 \%$ were failure cases, and $26.8 \%$ of patients died during treatment [10].

In this study, the majority of cases were new cases [93.7\%] and pulmonary TB [91.3\%], whereas retreatment and extra-pulmonary TB were $6.3 \%$ and $8.7 \%$ respectively.

The tuberculosis patients who were screed positive for HIV/AIDS were $86 \%$ less likely to have a successful TB treatment outcome than HIV/AID negative patients. This is the same to the study conducted in Woldie General Hospital that demonstrated Human Immunodeficiency Virus negative tuberculosis patients were 3.9 times more likely to have a successful treatment outcome than HIV positive tuberculosis patients and the study conducted to the Matema Hospital that showed co-infection with HIV were independent predictors for poor treatment outcomes [11]. This finding was similar to the study conducted in Gimbi and Afar region that demonstrated co-infection with HIV were independent predictors for poor treatment outcomes. [10]

The patients between the age group of $20-40$ were $83 \%$ less likely to have a successful TB treatment outcome than the patients with an age group of fewer than 20 years $[A O R ; 0.17,95 \% \mathrm{Cl}(0.04,0.76)]$. This finding is similar to a study conducted in the Bale Zone [12].

\section{Conclusion And Recommendation}

In the review of the document of 349 TB patients, $87.9 \%$ were successfully treated whereas $12.1 \%$ of the patients were unsuccessfully treated. From this, $53.4 \%$ and $34.5 \%$ of patients were cured and completed the treatment, respectively, whereas $3.7 \%, 5.8 \%$, and $2.6 \%$ of patients died, treatment failure, and defaulted respectively during the follow-up. The patients between the age group of $20-40$ were $83 \%$ less likely to have a successful TB treatment outcome than the patients with an age group of fewer than 20 years. The tuberculosis patients who were screed positive for HIV/AIDS had poor TB treatment outcomes. The patients who were registered for tuberculosis treatment in 2017 had four times successful TB treatment outcome than the patients who were registered in 2019. The proportion of patients with successful treatment outcomes was lower than the WHO target that was set to end TB strategy, which was $90 \%$ as well as no study, the prevalence of successful TB treatment outcome was $87 \%$. This finding was controversial than the national estimates of the Ethiopian ministry of health which indicates $96 \%$ of TB treatment success rate. Thus, public health facilities and the health department of Communicable Disease Control in the West Guji Zone may need to develop strategies to address new TB cases, while adhering to treatment and supporting health workers who work at health posts. Furthermore, it is recommended that these factors are studied using a strong study design and primary data 


\section{Limitation of the study}

The study was a cross-sectional in its design which makes it impossible to determine the causal relationship between TB treatment outcomes and associated factors. The document review measurements that were used might also have inherent biases and the potential for incompleteness of certain data.

Despite these limitations, the result of this study is important to give important clue for researches in similar population.

\section{Generalizability}

For this particularly study we have been employed the systematic sampling procedure. Thus, we can apply the result of this study to the general population.

\section{Declarations}

\section{Competing interests}

The authors declare that they have no financial or personal relationships that may have inappropriately influenced them in writing this article.

Funding: The authors received no financial support for the research, authorship, and/or publication of this article

\section{Availability of data and materials}

Author of this study declare that all data needed in this study can be available upon request by the side of corresponding author.

\section{Acknowledgements}

We would like to be thanks to all Bule Hora University, Bule Hora General Hospital, and West Guji Health department for their cooperation and willingness to provide the required information.

Also, we would like to thank my data collectors and Blue Hora general hospital staff for their unwavering support 
Finally, we would like to thank our participants for their cooperation and willingness to participate in the study.

\section{References}

1. Gebreegziabher B, Yimer S, Bjune G. Qualitative assessment of challenges in tuberculosis control in west Gojjam zone ,Ethiopia : health workers ' and tuberculosis control program coordinators ' perspectives. 2016 Sep.

2. Megerssa B, Yimer S, Gradmann C, Sagbakken M. Barriers for tuberculosis case finding in Southwest Ethiopia. 2020 Jan:1-20.

3. Feleke B, Alene D, Feleke E, Motebaynore Y, Biadglegne F. Clinical response of tuberculosis patients. 2018 Jul:1-11.

4. Daniel T. Treatment Outcomes of Tuberculosis Patients in Metema Hospital, Northwest Ethiopia. Mycobact Dis. 2014 Feb:5(4).

5. GUIDELINES FOR MANAGEMENT OF TB, DR-TB AND LEPROSY IN ETHIOPIA. Addis Ababa SIXTH EDITION .November 2017.

6. Vasankari T, Holmström P, Ollgren J, Liippo K, Kokki M and Ruutu P. Risk factors for poor tuberculosis treatment outcome in Finland. BMC Public Health. 2007 Aug: 7(291)

7. Ai .X, Men. K, Guo. L et al. Factors associated with low cure rate of tuberculosis in remote poor areas of Shaanxi Province, China. BMC Public Health.2010 Oct: 10(112)

8. Akhtar S, Rozi S, White F, Hasan R. Cohort analysis of directly observed treatment outcomes for tuberculosis patients in urban Pakistan. Internatinal Journal TB Lung Disease. 2011 Mar;15(1):90-6.

9. Tekkel M, Rahu H, Loit M, and Baburin A. Risk factors for pulmonary tuberculosis in Estonia. International Journal of Tuberculosis and Lung Disease.2002:6(10);887-894

10. Orofino R, Trajman C, Schmaltz M, Dalcolmo, and Rolla. Predictors of tuberculosis treatment outcomes. Jornal Brasileiro de Pneumologia.2012 Jun:38(1); 88-97

11. Hargreaves J, Kadzakumanja C, Whitt F, Salaniponi D, and S. B. Squire. Smear-negative pulmonary tuberculosis in a DOTS programme: poor outcomes in an area of high HIV seroprevalence. International Journal of Tuberculosis and Lung Disease.2001Feb:5(9); 847-854

12. Garedew D, Nemera G. Treatment outcome of tuberculosis and associated factors at gimbi town health facilities western oromia .2021 